\title{
Assessment of an automatic prosthetic elbow control strategy using residual limb motion for transhumeral amputated individuals with socket or osseointegrated prostheses
}

\author{
M. Merad, E. de Montalivet, M. Legrand, E. Mastinu, M. Ortiz-Catalan, A. Touillet, N. Martinet, J. Paysant, A.
} Roby-Brami and N. Jarrassé

\begin{abstract}
Most transhumeral amputated individuals deplore the lack of functionality of their prosthesis due to control-related limitations. Commercialized prosthetic elbows are controlled via myoelectric signals, yielding complex control schemes when users have to control an entire prosthetic limb. Limited control yields the development of compensatory strategies. An alternative control strategy associates residual limb motions to automatize the prosthetic elbow motion using a model of physiological shoulder/elbow synergies. Preliminary studies have shown that elbow motion could be predicted from residual limb kinematic measurements, but results with transhumeral amputated individuals were lacking. This study focuses on the experimental assessment of automatic prosthetic elbow control during a reaching task, compared to conventional myoelectric control, with six transhumeral amputated individuals, among whom, three had an osseointegrated device. Part of the recruited participants had an osseointegrated prosthetic device. The task was achieved within physiological precision errors with both control modes. Automatic elbow control reduced trunk compensations, and restored a physiologically-like shoulder/elbow movement synchronization. However, the kinematic assessment showed that amputation and prosthesis wear modifies the shoulder movements in comparison with physiological shoulder kinematics. Overall, participants described the automatic elbow control strategy as intuitive, and this work highlights the interest of automatized prosthetic elbow motion.
\end{abstract}

\section{INTRODUCTION}

Progress in mechatronics and robotics has facilitated the production of prostheses with an increasing number of active joints, like the Luke Arm for upper limb amputation [1]. Although the numerous degrees of freedom (DoFs) could enable a more human-like motion of the prosthesis, there has been a growing gap over the last decades between hardware improvements and control developments. Upper limb prosthetic users struggle to use modern devices, blaming various factors, such as phantom limb pain, socket discomfort or slippage, and counter-intuitive limiting control strategies. Myoelectric control has become for the last decades a common control

M. Merad, E. de Montalivet, M. Legrand, A. Roby-Brami and N. Jarrassé are with the CNRS, UMR 7222, ISIR / INSERM, U1150 Agathe-ISIR, Sorbonne University, Paris, France.

M. Ortiz Catalan and Enzo Mastinu are with the Biomechatronics and Neurorehabilitation Laboratory (@ChalmersBNL) at the Department of Electrical Engineering, Chalmers University of Technology, Gothenburg, Sweden.

A. Touillet, N. Martinet and J. Paysant are with the Institut Régional de Réadaptation, UGECAM Nord-est, Nancy France. method of prosthetic end-effectors [2], [3]. Myoelectric control derives from the residual limb's muscular electrical activity that is measured with surface electrodes (generally two) placed inside the prosthetic socket. The control scheme associates residual muscles' contractions to a prosthetic movement: for instance, residual biceps contractions control the prosthetic hand closing, and residual triceps contractions control the hand opening. Since all the myoelectrically-driven prosthetic joints are being controlled by the same two residual muscles, the user needs to switch in between prosthetic joints to be able to control one joint after the other, yielding a sequential control pattern, for instance controlling wrist rotation, then hand opening. Hence, as the number of prosthetic joints increases with the level of amputation, the overall control strategy becomes more difficult with only two control inputs. That is why most transhumeral amputated individuals are often fitted with only a myoelectric hand, and eventually a myoelectric wrist, but rarely with a myoelectric elbow, although commercially available, preferring a cable-driven or manuallylocked joint. The difficulty of controlling a prosthetic limb, especially for high amputation levels, causes the development of body compensatory strategies, with large trunk and shoulder displacements [4]. In addition to functionally impairing the user, such important modifications of the physiological behavior (i.e. movements without amputation) can lead to severe musculoskeletal disorders [5].

To overcome some of the limitations of conventional myoelectric control, pattern recognition approaches have been developed for over 40 years [6], aiming at a more precise decoding of myoelectric signals. These methods rely on finding distinct muscle activation patterns to control more types of movements using the same number of myoelectric inputs [7]. Pattern recognition-based control enables the utilization of several prosthetic movements without having a dedicated myoelectric signal to switch between the joints (for instance co-contractions or changes in contraction intensity). This requires the use of multiple recording sites, a precise extraction of different signal characteristics, and a multidimensional classification architecture [8]. A surgical technique, referred to as Targeted Muscle Reinnervation (TMR), increases the number of recording sites by rerouting amputated nerve branches (for instance brachial plexus nerves) to other muscles (for instance chest muscles) enabling vol- 
untary contractions of these newly reinnervated muscles [9]. Combining pattern recognition-based techniques to TMR can enable simultaneous control of several prosthetic joints [10]. While they are extensively studied in research applications, pattern recognition-based methods, like the COAPT system (http://www.coaptengineering.com/), have been only recently applied to commercialized prostheses. This delay can be explained by the numerous limitations of myoelectric signals. Indeed, the sensitivity to electrode shift, perturbations like muscle fatigue, or sweat [8] leads to a major robustness issue that is still to be addressed in current systems.

Alternative control inputs have been investigated in the literature, such as the contraction-induced skin vibrations, the contraction-induced skin deformation [11], with results that are not superior to myoelectric results. There have been investigations on the residual limb motion as a promising source of control inputs for prosthetic joints control. Indeed, most transhumeral amputated individuals have a preserved shoulder mobility, that is constrained for now by a harness. Some studies worked on a control strategy based on the shoulder joint mobility in the horizontal or vertical plane to drive the end-effector action [12], however it requires voluntary translation shoulder movements to control the prosthesis, like myoelectric control requires voluntary muscle contractions. Therefore, there is still a need for simultaneous and easy control strategy over artificial joints.

Upper limb motor control consists mostly in focusing on the task and the hand motion, while none or few of the attention is given to the individual control of each muscle or joints. The result is a coordinated movement of the joints along the upper limb, also know as a synergy for a given task. For instance, shoulder and elbow extends simultaneously and without reaching the individual's awareness while reaching for a target [13], [14], [15]. Several studies have shown that these synergies can be modeled, and thus, used to derive distal joint movements from measurements of proximal joints kinematics [16], [17], [18]. The study by Kaliki et al. [17] showed that the elbow flexion angle and the forearm rotation angle could be predicted using offline measurements of three shoulder angles and two shoulder translations, and an artificial neural network-based model of the upper limb joints motion for a reaching task. Based on these literature results, preliminary work focused on developing a shoulder/elbow coordination model for the reaching task using physiological kinematic data. Good offline prediction results of the elbow kinematics were obtained in [19] using two shoulder angular velocity values as model inputs, showing that automatic prosthetic elbow control was possible.

Most of training data sets in the literature are recorded using camera-based motion capture systems, which are not compatible with the environment of prosthetic users. Using wearable kinematic sensors is fundamental in the development of prosthetics. A good option is Inertial Measurement Units (IMUs) that can be interfaced with the existing hardware, and that provide reliable position and velocity information. Some recent studies used IMUs to measure the shoulder motion, but the shoulder/elbow models were only tested offline [20], [21]. The only results to the authors' knowledge of online prosthesis control using a shoulder motion-based control strategy is described in Alshammary et al. [22] whereby the participants controlled a virtual prosthesis driven by a shoulder/elbow model based on real time measurements of the participants' shoulder movements. Based on these literature results, a preliminary study, described in [23], was designed with non-amputated participants wearing a prosthetic elbow prototype which was driven by the participants' shoulder motion. However, it showed the need for tests with amputated individuals, as the quality of the prototype's attachment to the arm was poor. In [24], a first test was thus conducted with one transhumeral amputated individual who performed successfully a reaching task with the prosthesis prototype that was automatically-driven by the participant's residual limb motion and a shoulder/elbow synergy model built from movement recordings of two non-amputated individuals. Despite the promising results of the literature and preliminary studies, there is still a lack of extensive experimental evaluation conducted on amputated individuals to assess the performance of a prosthetic elbow automatically-driven by the residual limb motion, based on a shoulder/elbow coordination model built from recordings of several physiological reaching movements.

In the present paper, the automatic elbow control strategy was tested on a reaching task with six individuals amputated at the transhumeral level, in comparison to a conventional myoelectric elbow control strategy. Among the participants, some were equipped with an osseointegrated device that did not require the use of a traditional harness to hold the prosthesis. The participants, the prosthesis prototype, the experiment protocol and the data analysis are described in Section II. The results, presented in Section III and discussed in Section IV, showed an increase in body compensations when participants used the conventional myoelectric control, whereas overall body movements with the automatically-driven elbow were similar to a physiological gesture in terms of precision error and body behavior.

\section{MAterial AND MEthods}

\section{A. Participants}

This work was carried out in accordance with the recommendations of the Université Paris Descartes ethic committee CERES, that had approved the protocol covering preliminary experiments at the Louis Pierquin Center (Institut Régional de Médecine Physique et de Réadaptation, IRR) in Nancy. In addition, the protocol was approved by the ethical committees of Västra Götalandsregionen in Sweden, to conduct the experiment with osseointegrated participants. All participants gave written informed consent in accordance with the Declaration of Helsinki. To be included into the trial, participants had to be transhumerally amputated, not to suffer any residual limb pain, to have a good residual limb mobility with a preserved brachial plexus, to be equipped for more than a month with a myoelectric end-effector, and to have a residual limb length and strength that allowed the participant to lift the prosthesis.

Fourteen participants without amputation took part in a preliminary study aimed at gathering physiological kinematic data of reaching gestures. The only inclusion criteria was a 


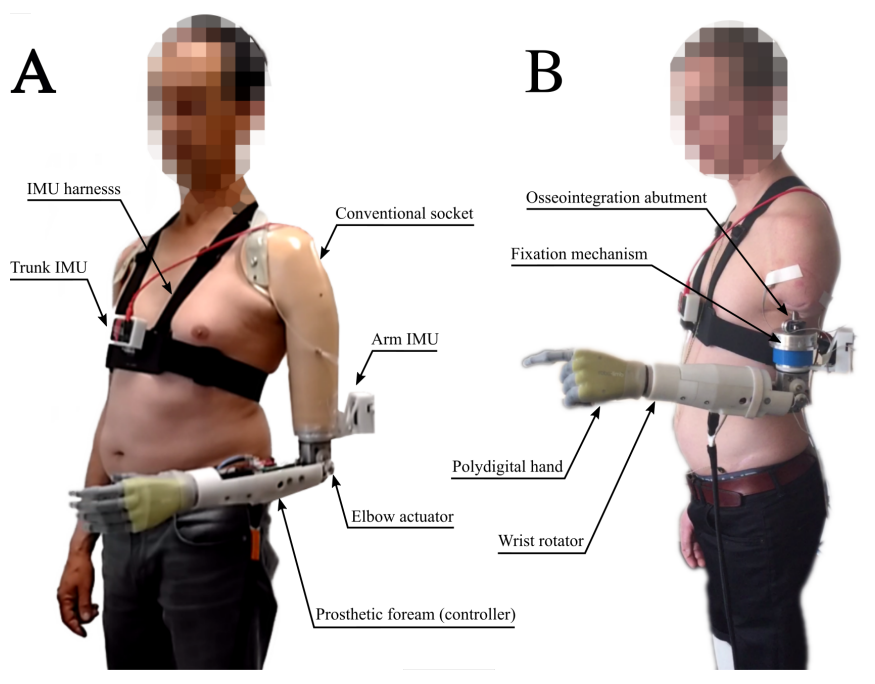

Fig. 1. A: amputated participant wearing the prosthesis prototype with a conventional external socket. B: amputated participant with the prosthesis prototype plugged to the metal bone rod implanted in the residual limb's bone. The prosthesis controller is connected, in addition to two myoelectric electrodes, to two IMUs placed on the chest and the socket, from which is derived the orientation of the arm/residual limb relatively to the trunk.

good physical condition. The average age was 24 years old $( \pm 2.1$ years old $)$, and the average height was $174 \mathrm{~cm}( \pm 10$ $\mathrm{cm})$. The data were used in the pre-experiment analysis, to build the shoulder/elbow synergy model to be implemented on the prototype for the experiment with the amputated individuals. Right and left arm gestures were not recorded the same day, and some participants did not come back to record the gestures with the other arm. In the end, we had the data from 10 non-amputated individuals to build the right model, and 10 individuals for the left model. The physiological kinematic data was also used in the post-experiment analysis for comparison with the reaching gestures performed by the amputated individuals.

Six amputated participants were recruited. Their own prosthetic equipment included a myoelectric hand, and for some of them a myoelectric wrist; therefore, all of them were already trained to do myoelectric control. They were split in two groups. The first group (Group Harness) was recruited at Centre Louis Pierquin in Nancy. Their own prosthetic equipment included a conventional external socket maintained by a harness, as illustrated in Fig. 1A. The second group of participants (Group Osseo) was recruited at the Biomechatronics and Neurorehabilitation Laboratory (Chalmers University of Technology, Gothenburg, Sweden), among participants of an ongoing experiment on osseointegrated prosthetic devices. These participants had undergone surgery consisting in inserting a titanium implant into their residual humerus bone [25]. Any prosthetic device can be fixed to the end of the percutaneous rod, without needing a harness to hold it, as illustrated in Fig. 1B. One participant with osseointegration controlled his own prosthesis with surface electrode, while the two others had been implanted recently with epimysial electrodes less than two months before the experiment [26] (e-OPRA implant system, Integrum AB, Sweden). Information on the amputated participants is provided in Table I.

\section{B. Prosthesis prototype}

Commercially available prosthetics components like a conventional electronic wrist rotator (model 10S17, Ottobock ${ }^{\circledR}$ ), and a modified E-TWO electric elbow (Hosmer, Fillauer ${ }^{\circledR}$ with a $10 \mathrm{~N} / \mathrm{m}$ of nominal torque and $50 \%$ of nominal speed) were assembled to form a two-DoF prosthetic forearm, as shown mounted on participants in Fig. 1. Any myoelectric prosthetic hand with the Quick Disconnect system could be interfaced with the prototype. During the experiment, left-amputated individuals used an i-Limb Ultra from Touch Bionics ${ }^{\odot}$ to perform the task, and right-amputated individuals used their own myoelectric hand (VariPlus Speed hand by Ottobock ${ }^{\odot}$ ) since we did not have a left i-Limb to provide to these participants at the time of the experiment. A Raspberry Pi 3 was used to read sensors, control the hand electronics and a dedicated motor controller in charge of elbow's and wrist's motor position and speed control. An encoder was added to the elbow motor for closed-loop control purpose. The forearm structure, in which most of the electronics was located, had been printed in ABS and reinforced with metal parts. The prosthetic forearm weighed $810 \mathrm{~g}$ without a prosthetic hand attached to it. The prosthesis prototype was mounted onto the subject's own socket, and their two myoelectric electrodes (Myobock, Ottobock ${ }^{\odot}$ ), located within their prosthesis socket over the residual biceps and triceps groups, were connected to the prototype's controller. For osseointegrated participants, the prototype was attached to the osseointegrated implant thanks to a specific adjustable mechanical part and the myoelectric signal measurements were read from a specific dedicated electronic interface which was included in the fixation mechanism for the two participants with implanted electrodes [27], and from standard myoelectric surface electrodes placed over the residual limb for the non-implanted one. The length difference between the limb equipped with the prosthesis and the physiological limb was was adjusted using spacers, and it was always less than $5 \mathrm{~cm}$.

The prosthesis controller read also the data from two IMUs (x-IMU, X-IO Technologies ${ }^{\odot}$ ), placed on the participant's trunk and arm. Finally, the controller piloted the prosthetic joints according to the input signals from IMUs and myoelectric electrodes, and the control mode in which the prosthesis was set. Indeed, two different control laws, described subsequently, were implemented on the prototype: the myoelectric control, based solely on myoelectric signals to control the prosthesis, and the automatic elbow control, driving the elbow joint based on IMU-based residual limb motion measurements and the shoulder/elbow synergy model implemented onto the controller.

\section{Myoelectric control (ME-mode)}

Although the prototype's joints were functional, the participants were asked to use only the elbow joint to perform the reaching task, since the study was focused on elbow control.

To prevent any involuntary control of the other prosthetic joints, for instance by unwillingly co-contracting muscles, the overall control algorithm was modified such that hand and wrist control was blocked during the experiment. Therefore, 


\begin{tabular}{|c|c|c|c|c|c|c|c|c|}
\hline & \multirow{2}{*}{$\begin{array}{c}\text { Gender } \\
\mathrm{M} / \mathrm{F}\end{array}$} & \multirow{2}{*}{ Age } & \multirow{2}{*}{ Height } & \multicolumn{2}{|c|}{ Amputation } & \multirow{2}{*}{ Osseo. ${ }^{\dagger}$} & \multirow{2}{*}{ EMG } & \multirow{2}{*}{ Myo.elbow? } \\
\hline & & & & Side & Date & & & \\
\hline S1 & $\mathrm{M}$ & 34 уо & $1 \mathrm{~m} 80$ & Left & 2014 & No & Surface & Yes \\
\hline S2 & M & 36 yo & $1 \mathrm{~m} 68$ & Left & 2017 & No & Surface & No \\
\hline S3 & M & 41 yo & $1 \mathrm{~m} 87$ & Right & 2015 & No & Surface & No \\
\hline $\mathrm{S} 4$ & M & 43 yo & $1 \mathrm{~m} 85$ & Left & 2011 & Yes & Implanted & No \\
\hline $\mathrm{S} 5^{*}$ & M & 28 yo & $1 \mathrm{~m} 75$ & Left & 2006 & Yes & Surface & No \\
\hline S6 & M & 42 yo & $1 \mathrm{~m} 87$ & Right & 1997 & Yes & Implanted & No \\
\hline
\end{tabular}

$\dagger$ Osseointegration.

* S5 had undergone bilateral transfemoral amputation, in addition to a left transhumeral amputation.

TABLE I

AMPUTATED PARTICIPANTS' GENERAL INFORMATION

participants could not switch to the hand or wrist control, enabling only elbow motion based on myoelectric signal. Since we relied on the participants' own electrodes (whether located in their socket or implanted), we used the myoelectric signal corresponding to prosthetic hand closing for elbow flexion, and the signal used for hand opening for the elbow extension. This resulted in a generic biceps/triceps activated control of the elbow flexion/extension. None of the participants had a myoelectric elbow in their own prosthetic equipment, except for Subject S1, who used only biceps contraction to bring the hand up, but had passive elbow extension. Hence, all the amputated participants were not familiar with motorized elbow motion nor the proposed myoelectric control method of the elbow.

\section{Automatic elbow control (A-mode)}

Using the A-mode, residual limb motion drove automatically the prosthetic elbow extension. Real time computation of the elbow angular velocity was derived from IMUmeasured residual limb's angular velocities, and one of the two shoulder/elbow synergy model, depending on the amputation side. Shoulder/elbow synergy models were built before the experiments, using for each side the recordings of 10 participants' physiological gestures. The input of the shoulder/elbow synergy model was the shoulder motion with respect to the trunk motion. The latter was obtained using an IMU placed on the participant's trunk (sternum). The arm IMU (respectively the trunk IMU) provided at each time a quaternion value representing the orientation of the arm (resp. the trunk), with respect to a position of reference. The relative orientation of the arm with respect to the trunk was calculated using the two quaternion values and transformation matrices to obtain three Euler angles in the ZYX sequence. Euler representation is commonly used in shoulder/elbow synergy modeling [16], [17], [28]. The result was then derived with respect to time to obtain the final model inputs, i.e. the three shoulder angular velocities with respect to the trunk motion. The model output was the elbow flexion/extension angular velocity. The shoulder/elbow synergy was modeled using a RBFN-based regression method as a linear combination of Gaussian functions [29], such that

$$
\dot{\beta}=\sum_{e=1}^{E} w_{e} \cdot \phi\left(\mathbf{x}, \theta_{e}\right)
$$

with $\dot{\beta}$ the elbow angular velocity, $\mathbf{x}$ the time derivatives of the three Euler angles describing the orientation of the arm with respect to the trunk, $\phi$ the radial basis functions (Gaussian functions), $w_{e}$ the weight for each function, and $\theta_{e}$ the $\mathrm{e}^{\text {th }}$ Gaussian basis function's radius.

The RBFN model was trained (which meant that the $w_{e}$ and $\theta_{e}$ parameters were obtained) with the data from 10 nonamputated participants, who repetitively performed the reaching gestures, following the same protocol as the amputated participants, which is described subsequently. Shoulder data were measured with the same IMUs sensors (trunk and arm) used with amputated participants, whereas the elbow angle was obtained with camera-based motion capture sensors. Only reaching phases were used to train the models; the return phases were not included. Further information on physiological data acquisition and design of the regression model is provided in [30].

\section{E. Experimental setup}

The overall experimental setup is illustrated in Fig. 2 A. The IMUs were placed on the participants' chest and arm/socket. A camera-based motion capture system, only used for off-line data analysis, recorded the participants' upper body kinematics at a frequency of $100 \mathrm{~Hz}$ : a Vicon@system (Vicon Motion System, Ltd.) was used with participants from IRR while a Codamotion@system (Charnwood Dynamics, Ltd.) was used with the osseointegrated participants. The main markers locations for both motion capture systems were: index's middle phalanx, hand's back, forearm, elbow lateral epicondyle, upper arm, both acromions, suprasternal notch, xiphoid process and both anterosuperior iliac spines.

The participants were asked to reach the 18 targets located in front of them. The targets were $4 \mathrm{~cm}$-yellow disks attached to three metal rods, placed at two distances (I, II), as illustrated in Fig. 2. They were numbered from 1 to 9 for each distance. The targets' positions were adjusted for each subject depending on their arm length and shoulder height. Target 8 was aligned with the subject's shoulder height in the sagittal plane (left shoulder if the task was performed with the left limb, right shoulder if performed with the right limb). Distance I was defined as the arm length minus $10 \mathrm{~cm}$, and Distance II corresponded to Distance I minus $15 \mathrm{~cm}$, as shown in Fig. II.2. The distance between the center and lateral targets, i.e. between Targets 1 and 2, or 2 and 3, was arbitrary fixed to 30 $\mathrm{cm}$. 


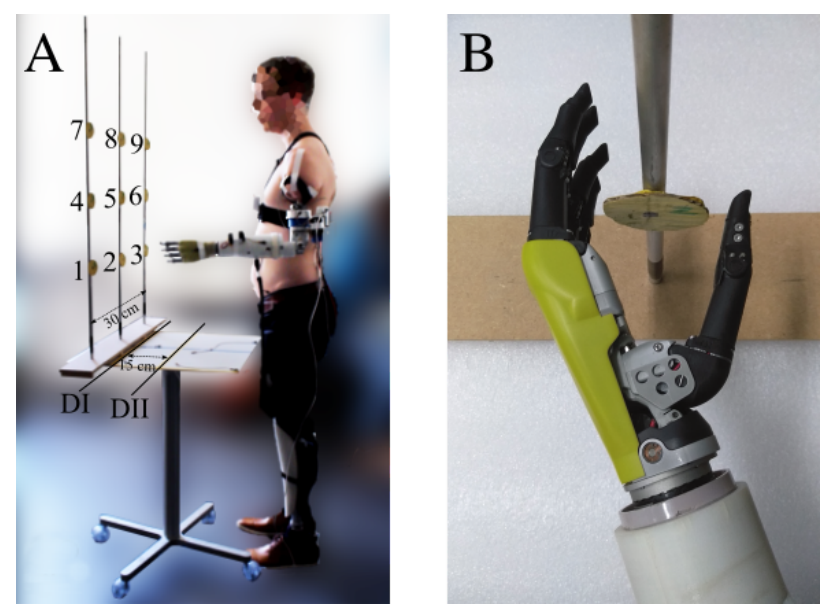

Fig. 2. Experimental setup. A: osseointegrated amputated participant wearing the prototype and standing in the initial position; there are 9 targets for each distance (I and II). B: Hand position with respect to the target when successfully performing the reaching task.

\section{F. Protocol}

Participants were fitted with the prosthesis prototype with the prosthetic hand placed in a semi-opened posture and a fixed orientation (semi pronated). They were asked to reach the different targets by trying to place the prosthetic hand around the different targets (as shown in Fig. 2 B). The task was achieved if the target was within grasp of the prosthetic hand. The initial position was defined with the prosthetic elbow flexed at 90 degrees, as shown in Fig. 2A. Even though hand and wrist could be myoelectrically-controlled, only prosthetic elbow motion was enabled by the control algorithm in both control modes during the experiment. For each pointing movement, the subjects stayed immobile in the initial position until told the target number to reach, then brought the prosthetic hand the closest as possible to the target, and stayed immobile until the elbow returned automatically (triggered by the experimenter) in a rigid control mode to the 90 degree-starting position. No particular instruction was given to the subjects concerning prosthetic joint use, movement strategy, duration, or speed.

The participants were instructed to do the main reaching movement in one action, as they would have done with their non-amputated limb. A one-action movement was defined based on usual concepts in human motor control, whereby a goal-directed physiological movement is characterized by a roughly linear end-effector's trajectory with a bell-shaped velocity profile, and a synergistic organization of joint coordination. Any perturbation in the sensorimotor context of movement execution, including using a prosthesis, can lead to a disruption of this picture. In this study, amputated participants were given the possibility with the A-mode to correct the end-effector's position with additional movements if the target was not reached at the end of the main reaching movement. This was enabled by a joint-locking feature that blocked the elbow once the main reaching movement was achieved, i.e. when the prosthesis controller detected an absence of motion from the residual limb. Therefore, the participants could move the residual limb without extending further the prosthetic elbow. These corrections of the end-effector's final position are referred subsequently to adjustment movements.

Participants performed the task once with the prosthetic elbow driven successively by each of the two control modes: during the first session (18 targets, i.e. 9 targets at two distances), the ME-mode (conventional dual-site myoelectric control strategy) was used, then, during the second session (18 targets), the participants used the A-mode (automatic elbow control strategy). Before the beginning of each session, the participants had 5 minutes to train with the control mode that was about to be tested. This training period was also used to provide additional instructions if the protocol was not understood. Also, they were asked not to move their feet during the reaching gestures.

\section{G. Data analysis}

1) Data processing: Data from the different sensors, such as the IMUs and the camera-based motion capture sensors, were collected and synchronized after the experiments. Data segments for each movement were cropped, using an arbitrary (individually chosen) threshold on the end-effector's velocity to detect the onset and end of movement, such that each obtained segment corresponded to a reaching movement towards a target. All cropped data segments were video-checked to make sure that analyzed data corresponded to the full reaching gesture.

2) Metrics: The performance was assessed with the final precision error, i.e. the distance between the hand's back marker and the target, and the completion time, i.e. time between movement onset and end. Because several participants used the elbow-locking feature with the A-mode to adjust the end-effector's final position, an intermediary calculation of the precision error (referred to as "precision error before adjustment movements" considered the reaching movement without the adjustment movement in order to depict the actual precision performance of the A-mode. The analysis was focused on comparing trunk, shoulder and elbow movements between the two prosthesis control modes, and also with the physiological participants' reaching movements. Several metrics assessed the trunk movements, including the angle ranges of the anteroposterior and mediolateral bending angles, and of the torsion angle. The trunk mean speed value corresponded to the trunk's cumulative trajectory (added displacements of the sternum's position throughout the movement) normalized by the completion time. The shoulder movements were assessed with the angle range of the humerus elevation angle, calculated as the angle between the humerus longitudinal axis and the trunk vertical axis. The elbow movement was assessed with the range of the flexion/extension angle. Adjustment movements were included in data segments for body kinematic analysis.

The overall synergy between shoulder and elbow movements was assessed using a method described in [31]. The synergy between the shoulder motion, represented by the humerus elevation angular velocity, and the elbow motion, represented by the elbow extension angular velocity, was compared to a synergy of reference. The latter was built from the physiological individuals' data set of reaching movements: a Principal 
Component Analysis (PCA) was run on the shoulder/elbow kinematic data to obtain the first Principal Component (PC), which represented the physiological synergy. PCAs were also performed on shoulder/elbow kinematic data of amputated participants for reaching gestures with the ME-mode and the A-mode. The comparison between the physiological synergy and the obtained synergy for the ME-mode and the A-mode was obtained by calculating for each control mode the angle between the physiological synergy and the first PC of the PCA on amputated participants' data. The more the two compared synergies differ, the larger the angle between the two first PC is. A similar analysis was performed by adding the trunk kinematics (represented by the trunk bending angle in the anteroposterior direction), yielding a comparison between trunk/shoulder/elbow synergies of physiological individuals and amputated individuals.
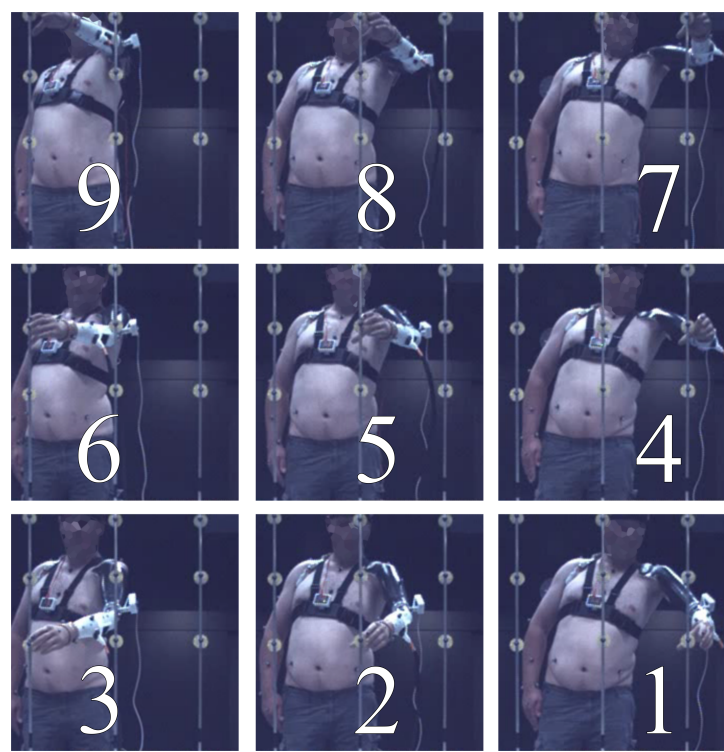

Fig. 3. Example of a trial performed by Subject S3 (with conventional socket). Depicted numbers correspond to the reached target for each of the 9 movements.

3) Statistical analysis: Repeated measures ANOVAs were carried out in the participants with the Group (Harness or Osseo) as between-subject factor, and the Mode (myoelectric or automatic) and the Target as within-subject factors. When there were significant interactions, two factors ANOVA (with Group as between-subject factor and Mode as within-subject factor) was performed separately for each target.

\section{RESULTS}

All participants could achieve the task with both control modes. An example of one trial performed by Subject S3 (Group Harness) with the ME-mode is depicted in Fig. 3. The A-mode, was appreciated by all the participants who described it verbally as an intuitive control method.

Despite the fact that the same instructions were given to all the participants, different reaching strategies could be observed between participants and control modes. Most participants positioned the elbow before reaching for the target with the ME-mode, achieving the reaching movement afterwards

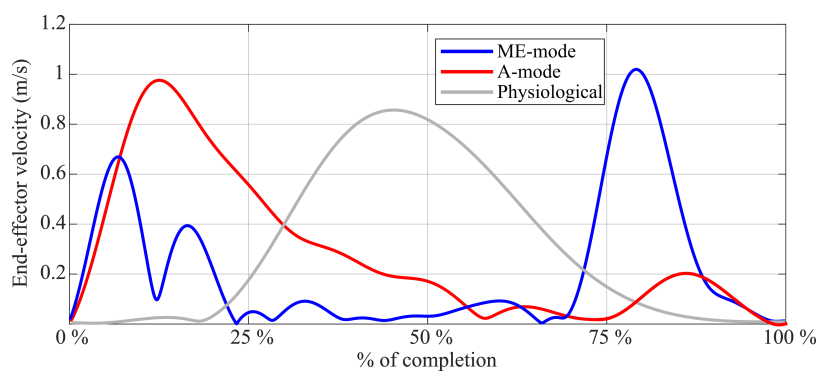

Fig. 4. Example of filtered end-effector velocity profiles, calculated as the norm of cartesian velocity. Blue (resp. red) line represents the velocity profile of Subject 4's reaching gesture towards Target I.8 with the ME-mode (resp. A-mode). The grey line represent the velocity profile of a non-amputated individual's reaching gesture towards the same target.

with permanent visual feedback and body compensations. In opposition, some participants used the elbow-locking feature with the A-mode to position the end-effector closer to the target, yielding a 2-step action composed of one main movement followed by a small final correction movement. Figure 4 depicts the velocity profiles of the end-effector for the two control modes for one amputated participant, and a physiological velocity profile. With the ME-mode, most participants positioned the prosthetic elbow (first blue peak) before performing the reaching movement by moving only the residual limb and the trunk (second blue peak). With the Amode, participants performed directly the reaching movement by simultaneously moving the shoulder and the elbow (first red peak), and some performed a final adjustment movement by moving the residual limb to reduce the distance to the target error (second red peak). However, most participants stopped their motion after the main reaching movement as they were close to the target.

\section{A. Functional assessment}

Final precision error: The experiment was focused on assessing the functionality of the tested automatic elbow control strategy. The reaching task is a functional task, and as the experiment was designed, achieving the task enabled variability on the final end-effector's position. Therefore, the task was considered as a success even if the precision error was not null. Variability can be seen with the precision error values from non-amputated participants who had an overall standard deviation of $38 \mathrm{~mm}$. Indeed, the results show that amputated participants had a precision error within the range of non-amputated participants in the two control modes, with an overall error of $1.5 \mathrm{~cm} \pm 1.3 \mathrm{~cm}$ with the ME-mode and $1.7 \mathrm{~cm} \pm 1.8 \mathrm{~cm}$ with the A-mode, in comparison with an average precision error of $1.4 \mathrm{~cm} \pm 1.9 \mathrm{~cm}$. Results are in Table II. The statistical analysis showed that the precision error did not vary significantly with the Mode, nor the Group. There were a borderline effect of the Target $(p=0.057)$. There were interactions between the effects of the Target and the Mode $(F(17,51)=1.95, p<0,027)$. However, these good precision results do not reflect the fact that some participants had to correct the end-effector's final position with the A-mode. Therefore, adjustments were removed from the performance analysis. 


\begin{tabular}{|c|c|c|c|c|c|c|c|}
\hline & \multicolumn{2}{|c|}{ Group Harness } & \multicolumn{2}{|c|}{ Group Osseo } & \multicolumn{2}{|c|}{ Amputated individuals } & \multirow{2}{*}{ Physiological } \\
\hline & ME-mode & A-mode & ME-mode & A-mode & ME-mode & A-mode & \\
\hline Final precision error $(\mathrm{cm})$ & $1.2 \pm 0.8$ & $1.7 \pm 1.6$ & $1.7 \pm 1.6$ & $1.6 \pm 2.0$ & $1.5 \pm 1.3$ & $1.7 \pm 1.8$ & $1.4 \pm 1.9$ \\
\hline Precision err. before adj. $(\mathrm{cm})^{*}$ & $1.2 \pm 0.8$ & $1.7 \pm 1.6$ & $1.7 \pm 1.6$ & $7.0 \pm 7.2$ & $1.5 \pm 1.3$ & $4.3 \pm 5.8$ & $1.4 \pm 1.9$ \\
\hline Completion time $(\mathrm{s})$ & $3.5 \pm 2.5$ & $2.3 \pm 1.1$ & $3.8 \pm 2.2$ & $2.5 \pm 1.5$ & $3.3 \pm 1.9$ & $2.4 \pm 1.2$ & $1.1 \pm 0.2$ \\
\hline Elbow range. (deg) & $18.3 \pm 21.1$ & $40.1 \pm 14.8$ & $16 \pm 18.4$ & $46.3 \pm 15.7$ & $17.1 \pm 19.7$ & $43.2 \pm 15.2$ & $34.9 \pm 10.1$ \\
\hline Humerus elev. (range deg) & $26.9 \pm 19.3$ & $33.3 \pm 16.5$ & $13.3 \pm 13.3$ & $23.2 \pm 17.2$ & $20.1 \pm 16.3$ & $28.2 \pm 16.8$ & $23.1 \pm 11$ \\
\hline Trunk mean speed $(\mathrm{mm} / \mathrm{s})$ & $38.9 \pm 20.7$ & $35.7 \pm 14.1$ & $32.0 \pm 31.3$ & $20.9 \pm 14.3$ & $35.4 \pm 26$ & $28.3 \pm 14.2$ & $14.0 \pm 11.1$ \\
\hline S/E coord. ${ }^{* *}(\mathrm{deg})$ & $27.7 \pm 12.7$ & $4.5 \pm 2.8$ & $41.7 \pm 11.2$ & $21.3 \pm 5.8$ & $34.7 \pm 11.9$ & $12.9 \pm 4.3$ & $\mathrm{X}$ \\
\hline T/S/E coord. ${ }^{* * *}(\mathrm{deg})$ & $35.0 \pm 4.2$ & $24.0 \pm 8.7$ & $51.9 \pm 7.8$ & $25.6 \pm 11.7$ & $43.4 \pm 6$ & $24.8 \pm 10.2$ & $\mathrm{X}$ \\
\hline
\end{tabular}

* With the A-mode, precision error are calculated before adjustment movements.

** S/E coord. denotes the difference between the shoulder/elbow coordinations of physiological and amputated individuals.

*** T/S/E coord. denotes the difference between the trunk/shoulder/elbow coordinations of physiological and amputated individuals.

TABLE II

RESULTS OF THE KINEMATIC FEATURES FROM THE REACHING TASK WITH AMPUTATED PARTICIPANTS, COMPARED WITH A PHYSIOLOGICAL REFERENCE.

Precision error before adjustment movements: All the participants could achieve the task with both control modes by placing the hand around the target with a precision error similar to the physiological controls. Precision errors with the A-mode were also computed without adjustments movements if there were any in the considered reaching movement. The corresponding values are depicted in Fig. 5A. Larger values indicate that adjustment movements were sometimes necessary to achieve the task. The overall precision error values, averaged over all targets, distances and participants of each group, are reported in Table II. The overall precision error for amputated participants was $1.5 \mathrm{~cm} \pm 1.3 \mathrm{~cm}$ using the MEmode, and $4.3 \mathrm{~cm} \pm 5.8 \mathrm{~cm}$ using the A-mode. Without a clear reason, participants of the Group Osseo used more frequently a two-step strategy with the A-mode, with large adjustment movements, that lead to a larger overall precision error of 7.0 $\mathrm{cm} \pm 7.2 \mathrm{~cm}$. The statistical analysis showed that the precision error varied with the Target $(F(17,51)=3.71, p<0.0001)$, with a borderline effect of the Group $(p=0.051)$ and the Mode $(\mathrm{p}=0.06)$. There were strong interactions between the effects of the Target and the Group $(F(17,51)=2.73, p<0.005)$, the Target and the Mode $(F(17,51)=2.72, p<0.005)$. There were no significant result but borderline tendencies (between 0.05 and 0.08) for the effect of the Group (Targets I.1-3, II.8), the Mode (Targets I.1, I.3, I.7).

Completion time: The reaching gestures performed with a prosthesis were longer $(3.3 \mathrm{~s} \pm 1.9 \mathrm{~s}$ with the ME-mode, and $2.4 \mathrm{~s} \pm 1.2 \mathrm{~s}$ for A-mode) than physiological movements (1.1 $\mathrm{s} \pm 0.2 \mathrm{~s}$ ), as shown in Fig. 5B. However, the completion time was reduced using the A-mode, as shown by the completion time values grouped in Table II. The statistical analysis showed that the completion time did not vary significantly, with a borderline effect of the Mode $(\mathrm{p}=0.060)$, and the Target (0.066).

\section{B. Movement strategy assessment}

A typical reaching movement is illustrated in Fig. 6. These pictures represent the initial and final postures taken by Subject S6 while he performed the reaching movement towards Target 5 of Distance I with the prosthetic elbow in ME-mode, and in A-mode. It appeared that the participants' overall motor strategy was different depending on the prosthetic elbow's control mode. Indeed, differences in the trunk kinematics could
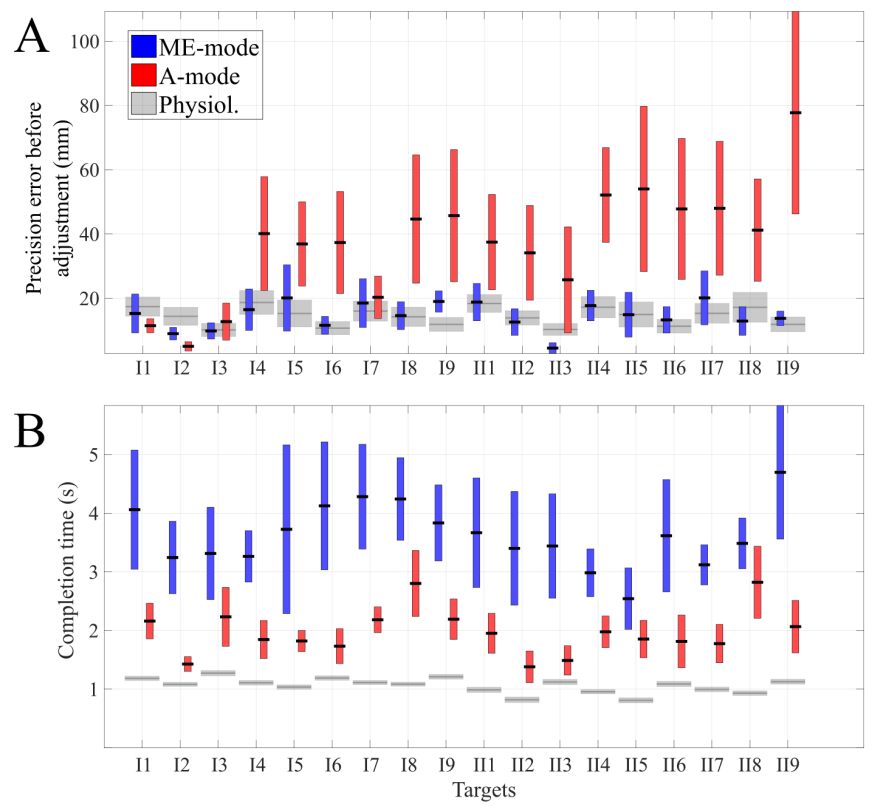

Fig. 5. Precision errors (A) and task completion times (B) with the MEmode (blue bars) and the A-mode (red bars) for all targets. Small green lines represent the mean value, and bars represent the standard deviation. Values are calculated before adjustment movements. Grey lines and shaded areas represent the averaged precision errors and completion times, and the corresponding standard deviation, of averaged physiological reaching movements.

be observed between the two trials. The results were compared to an average of physiological reaching strategies.

Elbow joint utilization: Depending on the control mode, the participants had a different use of the prosthetic elbow. The elbow angle ranges were averaged over the Group. They are depicted in Fig. 7A. When compared to a physiological elbow angle ranges $(34.9 \mathrm{deg} \pm 10.1 \mathrm{deg})$, it seems that the A-mode allowed a greater involvement of the elbow into the task (43.2 deg $\pm 15.2 \mathrm{deg})$, whereas the prosthetic elbow utilization was more limited with the ME-mode (17.1 deg $\pm 19.7 \mathrm{deg}$ ). However there were not a distinct difference between the groups of amputated participants, as shown in Table II. The statistical analysis showed that the elbow angle ranges varied with the control Mode $(p<0.05)$, especially for targets located at Distance II (Targets I.4, I.8, II.4-9). 

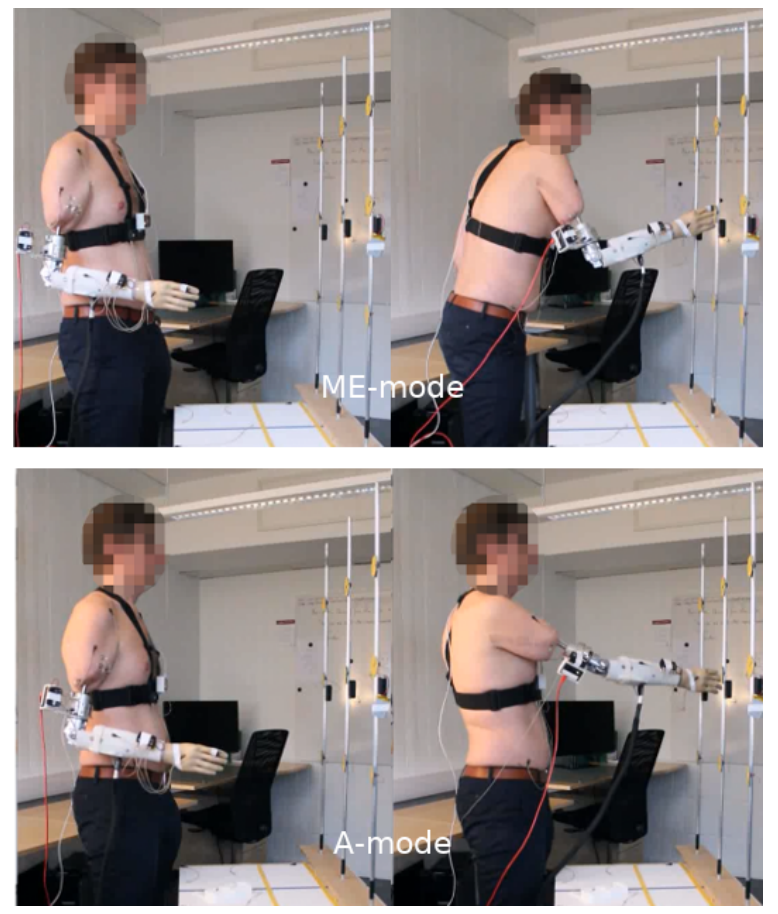

Fig. 6. Reaching movements with initial and final postures towards Target 5 (Distance I) with the ME-mode (left) and with the A-mode (right).
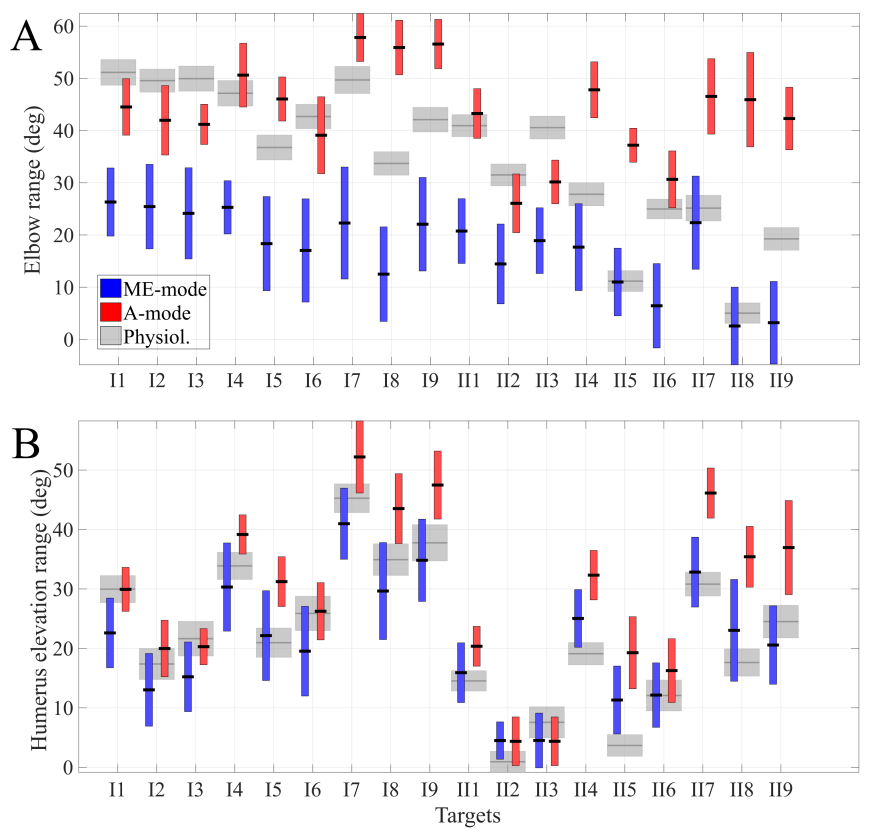

Fig. 7. Depiction of the elbow angle ranges, i.e. the difference between final and initial elbow angle values for each target, averaged over the 6 amputated participants for the two control conditions (ME-mode in blue and A-mode in red), and compared to the averaged reaching strategies of physiological individuals (grey bars). Green lines represent the mean value, and bars represent the standard deviation. B: Humerus elevation angle ranges represent the residual limb motions for each target.

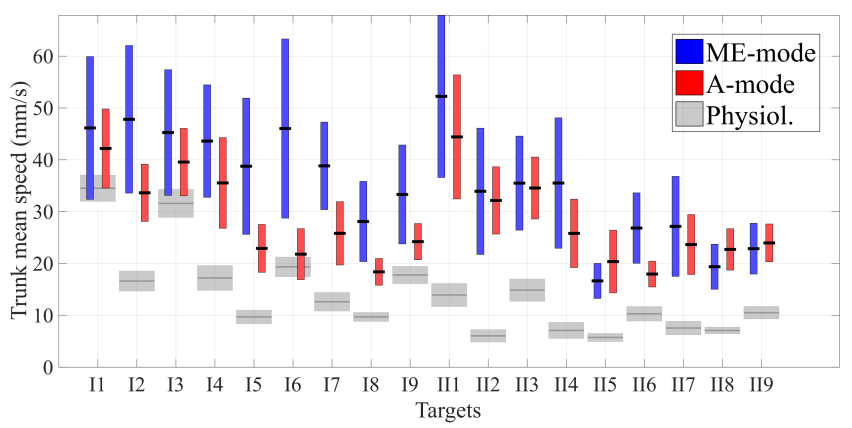

Fig. 8. Averaged trunk mean speed (i.e. trunk cumulative trajectory normalized by the completion time) and their corresponding standard deviations are represented by green lines and blue bars (ME-mode) and red bars (A-mode).

Shoulder joint utilization: Averaged humerus elevation angle ranges are depicted in Fig. 7B. Moreover, values are shown in Table II. Results tend to show that the A-mode increases the humerus elevation range, especially for participants in the Group Osseo. Nonetheless, differences of the two control modes with respect to physiological values remain limited (20.1 deg $\pm 16.2 \mathrm{deg}$ for ME-Mode, $28.2 \mathrm{deg} \pm$ $16.8 \mathrm{deg}$ for A-mode, whereas the physiological elbow angle range was $23.1 \mathrm{deg}+/-11.1 \mathrm{deg}$ in average. The statistical analysis showed that the humerus elevation angle range varied significantly $(p<0.05)$ with the control mode only for Targets II.7 and II.9).

Trunk movements: The prosthetic elbow in ME-mode was generally used to position the forearm before the actual reaching motion. The end-effector was then brought to the targets by elevating the humerus, and in some cases, by leaning over the table, yielding large body displacements. The trunk mean speeds were larger for movements performed with the MEmode, as depicted in Fig. 8. Specifically, trunk displacements of Group Osseo's participants were largely reduced with the A-mode, whereas they were still important in Group Socket, as shown by the overall trunk mean speed values in Table II. The average trunk mean speed was $25.4 \mathrm{~mm} / \mathrm{s} \pm 26 \mathrm{~mm} / \mathrm{s}$ with the ME-mode, and $28.3 \mathrm{~mm} / \mathrm{s} \pm 14.2 \mathrm{~mm} / \mathrm{s}$ with the Amode, compared to $14 \mathrm{~mm} / \mathrm{s} \pm 11.1 \mathrm{~mm} / \mathrm{s}$ in non-amputated individuals. The trunk mean speed varied significantly with the Target $(F(17,51)=4.4, p<0.0001)$ without significant interactions.

The analysis of trunk movements showed different reaching strategies depending on the target location and the control mode, especially movements in the anteroposterior direction and torsion movements, as shown in Fig. 9. The A-mode lead to a trunk backward motion for high-located targets (Targets I.7-9): it seems that it was mostly due to an an over-extension of the prosthetic elbow with the A-mode that was corrected by the participants by leaning their trunk backwards in order to reach these targets. Trunk torsion towards the contralateral limb was increased with the A-mode for participants in both groups.

Upper limb synergies: The upper limb synergies (shoulder/elbow and trunk/shoulder/elbow) of the amputated participants performing with the two control modes were compared to a physiological synergy. An illustrative example of the 

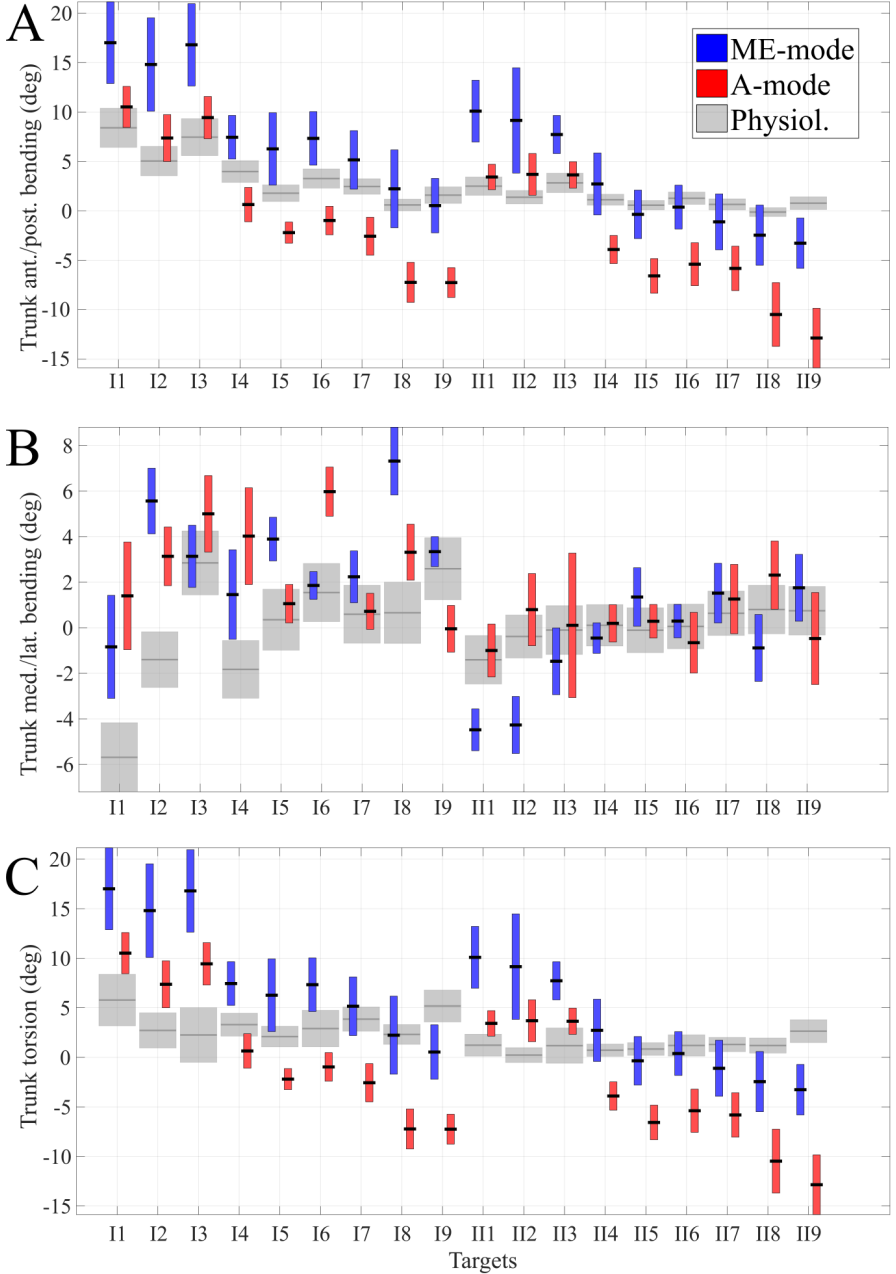

Fig. 9. Trunk angle ranges for anteroposterior movements (A), mediolateral movements (B) and torsion movements (C) for all targets. Short green lines and their corresponding bars represent the averaged range values and their corresponding standard deviation for amputated participants performing the task with the ME-mode (blue bars) and the A-mode (red bars).

comparison between the shoulder/elbow synergies is shown in Fig. 10: it highlights the desynchronization between shoulder and elbow movements with the ME-mode, while showing a better coordination between shoulder and elbow movements with the A-mode, closer to a physiological shoulder/elbow coordination. Averaged resulting values of the angle between the PCs are depicted in Table II. Using the A-mode reduced the difference between the upper limb synergies of physiological individuals and the amputated participants: an averaged angle of $34.7 \mathrm{deg} \pm 11.9$ deg was calculated with the ME-mode, and of $12.9 \mathrm{deg} \pm 4.3 \mathrm{deg}$ with the A-mode. The angles between the synergies increased when the trunk motion was added to the kinematic analysis of synergies: the averaged angle was $35.5 \mathrm{deg} \pm 4.2 \mathrm{deg}$ with the MEmode, and $24.8 \mathrm{deg} \pm 8.7 \mathrm{deg}$ with the A-mode. For the shoulder/elbow synergy, there were significant Target*Mode and Target*Group interactions $(F(17,51)=1.91, p<0.05$, and $F(17,51)=2.55, p<0.01$ respectively). There were borderline tendencies for the effect of Group (Targets I.24, II.1) and Mode (Targets I.2, II.7). The synergy between
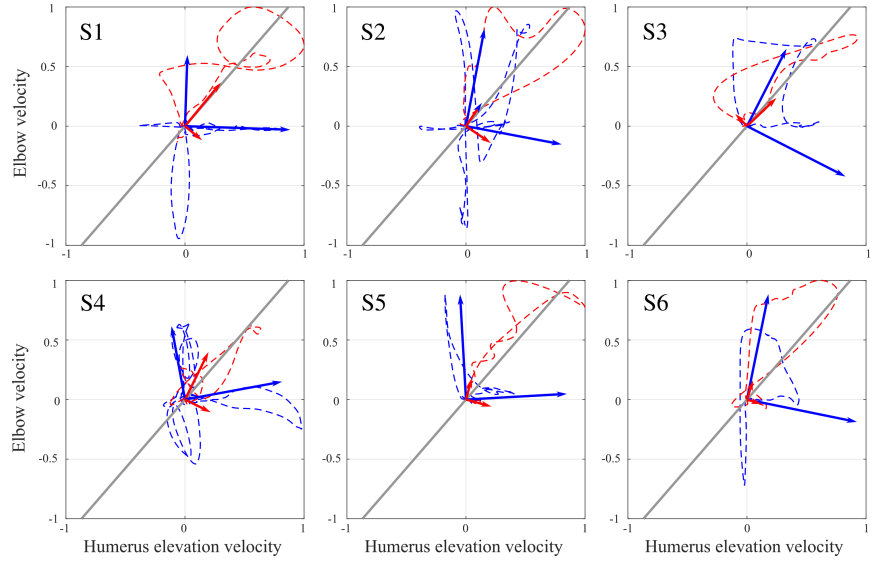

Fig. 10. Example of the shoulder/elbow synergies, expressed in terms of angular velocities of the humerus elevation angle and elbow flexion/extension angle, of the 6 amputated participants for reaching movement towards Target I.5 with the ME-mode (dashed blue line) and the A-mode (dashed red line). Blue and red vectors represent the first and second Principal Components (PCs) of the PCA performed on the shoulder/elbow angular velocities. The grey line represents the first PC of the PCA performed on physiological shoulder/elbow angular velocities. The shoulder/elbow synergy is assessed by calculating the angle between the grey line and the first PC of amputated participants' data for each control mode.

the trunk, shoulder and elbow movements varied significantly with the Mode $(F(17,51)=10.35, p<0.05)$ with significant Mode*Target*Group interactions $(F(17,51)=2.2, p<0.01)$. There was a significant Mode*Group interaction for Targets I.8 and II.6, and borderline tendencies for the effects of Group (Targets I.1-4, II.7, II.8), Mode (Targets II.2, II.4, II.8) and Mode*Group interactions (Targets I.4, I.7, II.4, II.9).

\section{DISCUSSION}

The results showed that the six amputated participants were able to perform the task with the two prosthetic elbow control modes. None of them was familiar with the imposed myoelectric strategy for the elbow joint, and most of them used to have only a myoelectric hand in their own prosthetic equipment. Although they tried the A-mode in a restrictive reaching task, the participants approved the concept by verbally describing it as more intuitive than myoelectric control. They indeed reported appreciating the fact of not being forced to anticipate the final posture of the arm and to pre-position the elbow accordingly before performing the reaching action: A-mode allowed them to rather stay focused on the end-effector only. The reaching gesture is not part of the daily gestures performed with a prosthesis, especially for transhumeral amputated individuals. For the presented experiment, participants tried for 5 minutes the task with the control mode they were about to test. The training period was thus too short to test all the targets, and neither to get used to the task. This time was used by the experimenters to make sure that they understood the task, and were able to use the system. Although all participants performed with the ME-mode first, the performed task was considered sufficiently short to avoid learning from one trial to another, as they reached only once for each target with each control mode. 


\section{A. Precision error}

Most participants could not perform the task with the MEmode by simultaneously extending the elbow via myoelectric control and moving the residual limb to reach for the target. They positioned myoelectrically the elbow before reaching for the target, making the overall strategy sequential and nonphysiological. Similar behaviors were observed in the studies in [4] and [32]: the elbow angle or the objects themselves were positioned before the reaching movement to make the task achievement easier. Nonetheless, positioning the prosthesis beforehand did not reduce the compensatory behavior, and neither reduced the completion time.

Contrary to the ME-mode, the A-mode enabled most of the participants to reach for the targets with a small error in one go. Specifically, participants in Group Harness achieved the task with the A-mode with a physiologically-like precision, whereas the averaged precision error (before adjustment) was larger in Group Osseo. The latter result was mainly due to one participant who extensively used the elbow-locking feature with the A-mode: he extended rapidly the elbow, and adjusted the end-effector position afterwards, yielding larger precision error. Giving more instruction on how to achieve the task with the A-mode could have avoided this phenomenon. However, this first experiment with several amputated individuals was also an opportunity for the experimenters to observe how amputated individuals intuitively interacted with the new control method. It appeared that several targets located at a closer distance were difficult to reach with the A-mode as they required a small elbow movement; it was especially the case for Targets II.7, II.8 and II.9, for which almost no elbow extension was required, but residual limb movement lead inevitably to an elbow extension, yielding a large error for these targets. Participants would have had to lock the elbow before moving the residual limb, which needed at least more training with the device to achieve this level of anticipation.

\section{B. Completion time}

Since the A-mode was based on simultaneous extension of the shoulder and the elbow, participants could only focus on bringing the end-effector to the target, yielding a shorter completion time. However, values were still longer than physiological baseline values, possibly due to a lack of training with the A-mode. Although the participants were familiar with myoelectric control, they struggled to perform the task with the ME-mode, mostly because they were not used to have a motorized elbow, neither to do myoelectric control while moving the residual limb. That is why they chose to position the prosthetic elbow before bringing the end-effector to the target. This positioning phase explains a longer completion time. Since the ME-mode required an elbow pre-positioning phase before the actual reaching movement, participants seemed to feel limited by the prosthesis. Thus, they tended to extend the elbow to a position that was roughly going to match the target, and they compensated with the upper body the lack of mobility of the prosthesis to reach the target.

\section{Analysis of body kinematics}

The analysis of the elbow joint's range of motion showed that the utilization was different from one control mode to another. Compared to the physiological baseline, amputated participants under-used the prosthetic elbow with the MEmode. Interestingly, some participants chose to flex the elbow to achieve the task towards some targets, especially highlocated targets, although no instruction was given on the expected reaching strategy. The A-mode appeared to restore a physiological utilization of the elbow joint with larger and more physiologically-like angle ranges, except for close or high-located targets. Effects on the humerus elevation were undetermined with both control modes: while participants elevated more the arm with the A-mode, it only had a visible effect in Group Osseo. This could be explained by a more physiological attachment of the prosthesis to the body, and thus, increased ranges of motion of the residual limb. The reaching strategy chosen by most participants with the ME-mode, consisting in positioning the elbow before moving towards the target, was the costliest in terms of trunk compensatory movements. Elbow extension performed in anticipation was often prematurely interrupted, yielding increased trunk forward bending motion to compensate for the lack of elbow extension. As a result, amputated participants had a significantly larger involvement of their trunk with the ME-mode than non-amputated individuals. In addition, elevating the residual limb with the prosthesis lead in some participants to involuntary residual limb muscle contractions, yielding reduced residual limb movements and larger trunk compensatory movements with the ME-mode.

Elbow impairment and the use of simple mechanical lockable elbow prosthesis evokes large trunk movements [4], [33], [34]. The study in [4] measured 35-cm trunk displacements in the anteroposterior and mediolateral directions, and a shoulder cumulative trajectory of $50 \mathrm{~cm}$ during reaching movements of transhumeral amputated individuals. Important modifications of the physiological behavior, as observed with some of the recruited participants in the present study with the ME-mode, can explain the occurence of musculoskeletal disorders in amputated individuals.

Body compensations were reduced with the A-mode for participants in both groups. However, elbow over-extension for high-located targets had an opposing effect: instead of trunk forward bending motion, large backward bending angle ranges were measured for some participants. In fact, the body was more involved in reaching movements of amputated participants, than with non-amputated participants. A possible explanation was the difficulty to lift the prosthesis weight above a certain height, requiring the utilization of the upper body. Even though the prototype's weight was similar to a commercialized prosthesis, attachment to the body (either osseointegrated or externally attached via a harness and external socket) lead to important non-physiological forces applied to the residual limb, already weakened by the amputation. 


\section{Upper limb synergies}

Reaching movements performed with the A-mode appeared to be closer to a physiological strategy, especially in terms of in-between joints synchronization. The A-mode seemed to restore the coordination between upper body joints, which was non-existent with the ME-mode, as shown by the endeffector's velocity profiles and the shoulder/elbow synergy analysis results.

The shoulder/elbow synergy model was implemented with the assumption that the residual limb kinematics were similar to the physiological shoulder kinematics included in the model training data set. As shown in a preliminary study [24], the residual limb movements and physiological shoulder movements are kinematically different. Limb loss affects the residual limb kinematics by altering the whole sensorimotor loop. The amputated participants, who were used to have a missing limb and a prosthesis, did not have time to internalize the proposed automatic prosthetic elbow control strategy. As a result, persistent acquired post-amputation motor control strategies could be observed in the residual limb movements. Hence, mobilizing the residual limb with a prosthesis requires training.

\section{E. Inter-individual variability}

A key observation is that amputation seems to be associated with an important inter-individual variability. As a results, the relevance of generalized analyses and methods should be discussed. Although the recruited participants were familiar with myoelectric control, they did not have the same hardware experience. Among the recruited participants, the time span between first myoelectric prosthetic equipment and the day of the experiment ranged from one month to 10 years. Thus, their performance with the ME-mode was possibly influenced by the expertise level with myoelectric control.

The prosthesis attachment to the body influenced also the participants' performance, particularly with the ME-mode. Participants for whom the prosthesis socket did not maintain a tight contact between the surface electrodes and the skin, encountered additional difficulty when they moved the residual limb: vibrations caused by the prosthetic elbow actuation or small movements inside the socket evoked signals artifacts that lead sometimes to undesired elbow extension with the ME-mode.

\section{F. Study limitations}

Participants were not familiar with performing Activities of the Daily Living (ADLs) with a motorized elbow. They had only 5 minutes to explore each control strategy, which was clearly not enough to be familiar with the proposed control solution.. Combining residual limb motion and myoelectric control evoked eventually involuntary muscle contractions of the residual limb, which could be avoided with a better prosthesis attachment to the body, with implanted electrodes, and with training. Despite the fact that the participants were satisfied with the presented automatic elbow control strategy, better results in terms of performance, and thus, in terms of body behavior, could be obtained with training of the participants. The shoulder/elbow synergy model was built as a synthesis of 10 different physiological shoulder/elbow synergies. By combining physiological data sets, the shoulder/elbow synergy model assimilates inter-individual variability, but remains different from the user's own reaching strategy. Thus, the paradigm whereby physiological shoulder/ebow synergies are driving a prosthetic elbow may not be adapted to prosthesis users. Although being encouraging in terms of compensatory movement reduction, the present results justifies for the need of a model that is tailored to the user's residual limb capabilities in terms of movement and control.

\section{G. Future work}

While this work was only focused on a simplified case of reaching movements, future work should be focused on adapting the automatic elbow control strategy to other ADLs, hence adding more gestures to the catalog of automated elbow movements. Thus, one could imagine a global control framework that switches between different shoulder/elbow synergy models depending on the task to be performed.

The automatic prosthetic elbow control strategy enables simultaneous elbow and end-effector control since the residual limb motion is used to drive the prosthetic elbow, and myoelectric signal are available to control the end-effector and the wrist. This feature was tested off experiment: one participant in Group Osseo and one in Group Harness were able to control their myoelectric hand while controlling simultaneously the elbow with residual limb motion, without any training. As simultaneous control is a sought feature in prosthetic control, future tests will explore the possibilities of the combined myoelectric and automatic control.

\section{CONCLusions}

Six individuals with transhumeral amputation achieved a reaching task with prosthetic elbow prototype driven by a shoulder/elbow synergy model, which was built from a combination of physiological reaching data. For comparison, the participants performed the task also with a conventional dualsite myoelectric elbow control strategy. This task, unusual for transhumeral amputated individuals, was achieved successfully with both control modes. In terms of body behavior, the automatic elbow control strategy restored the shoulder/elbow synergy, whereas shoulder and elbow movements were decomposed using myoelectric control. The participants verbally described the automatic elbow control strategy as intuitive. In a near future, such approach could allow simultaneous control of the elbow, via residual limb motion-based control, and of the prosthetic hand, via myoelectric control. While numerous challenges remain unsolved for the presented approach to be transferred to a commercialized device, this study showed the potential benefit of an automatic elbow control strategy.

\section{ACKNOWLEDGMENT}

The authors warmly thank the patients included in this protocol along with the orthopaedic-fitting team of the IRR (in particular F. Codemard, K. Georges Y. Corbel and B. 
Juncker) and the members of the Biomechatronics and Neurorehabilitation Laboratory (BNL) of Chalmers University of Technology. The study was financially supported by Sorbonne Université (project PROCOSY) as part of the Idex SUPER and the Labex SMART (ANR-11-LABX-65) supported by French state funds managed by the ANR within the Investissements d'Avenir programme under reference ANR-11-IDEX-0004-02. The participation of BNL was financially supported by the Promobilia Foundation, VINNOVA, and by Swedish Research Council (Vetenskapsrådet).

\section{REFERENCES}

[1] Linda Resnik, Shana L Klinger, and Katherine Etter. The DEKA Arm: Its features, functionality, and evolution during the veterans affairs study to optimize the DEKA Arm. Prosthet. Orthot. Int., 38(6):492-504, 2013.

[2] Peter J Kyberd and Wendy Hill. Survey of upper limb prosthesis users in sweden, the united kingdom and canada. Prosthetics and orthotics international, 35(2):234-241, 2011.

[3] Ning Jiang, Strahinja Dosen, Klaus-Robert Müller, and Dario Farina. Myoelectric control of artificial limbs-is there a need to change focus? IEEE Signal Process. Mag., 29(5):152-150, 2012.

[4] Anthony J Metzger, Alexander W Dromerick, Rahsaan J Holley, and Peter S Lum. Characterization of compensatory trunk movements during prosthetic upper limb reaching tasks. Arch. Phys. Med. Rehabil., 93(11):2029-2034, 2012.

[5] Kristin Østlie, Rosemary J Franklin, Ola H Skjeldal, Anders Skrondal, and Per Magnus. Musculoskeletal pain and overuse syndromes in adult acquired major upper-limb amputees. Arch. Phys. Med. Rehabil., 92(12):1967-1973, 2011

[6] F Ray Finley and Roy W Wirta. Myocoder studies of multiple myopotential response. Archives of physical medicine and rehabilitation, 48(11):598-601, 1967.

[7] Kevin Englehart and Bernard Hudgins. A robust, real-time control scheme for multifunction myoelectric control. IEEE Trans. Biomed. Eng., 50(7):848-854, 2003.

[8] Claudio Castellini, Panagiotis Artemiadis, Michael Wininger, Arash Ajoudani, Merkur Alimusaj, Antonio Bicchi, Barbara Caputo, William Craelius, Strahinja Dosen, Kevin Englehart, et al. Proceedings of the first workshop on peripheral machine interfaces: going beyond traditional surface electromyography. Front. Neurorobot., 8:1-17, 2014.

[9] Todd A Kuiken, Laura A Miller, Robert D Lipschutz, Blair A Lock, Kathy Stubblefield, Paul D Marasco, Ping Zhou, and Gregory A Dumanian. Targeted reinnervation for enhanced prosthetic arm function in a woman with a proximal amputation: a case study. The Lancet, 369:371-380, 2007.

[10] Todd A Kuiken, Guanglin Li, Blair A Lock, Robert D Lipschutz, Laura A Miller, Kathy A Stubblefield, and Kevin B Englehart. Targeted muscle reinnervation for real-time myoelectric control of multifunction artificial arms. JAMA, 301(6):619-628, 2009.

[11] Jorge Silva and Tom Chau. Coupled microphone-accelerometer sensor pair for dynamic noise reduction in mmg signal recording. Electron. Lett., 39(21):1-2, 2003.

[12] Yves Losier, Kevin Englehart, and Bernard Hudgins. Evaluation of shoulder complex motion-based input strategies for endpoint prostheticlimb control using dual-task paradigm. J. Rehabil. Res Dev., 48(6):669, 2011.

[13] JF Soechting and F Lacquaniti. Invariant characteristics of a pointing movement in man. J. Neurosci., 1(7):710-720, 1981.

[14] F. Lacquaniti, J. F. Soechting, and C. A. Terzuolo. Some factors pertinent to the organization and control of arm movements. Brain Research, 252(2):394-397, 1982.

[15] M. C. Cirstea, A. B. Mitnitski, A. G. Feldman, and M. F. Levin. Interjoint coordination dynamics during reaching in stroke. Experimental Brain Research, 151(3):289-300, 2003.

[16] Mirjana Popović and Dejan Popović. Cloning biological synergies improves control of elbow neuroprostheses. IEEE Eng. Med. Biol. Mag., 20(1):74-81, 2001.

[17] Rahul R Kaliki, Rahman Davoodi, and Gerald E Loeb. Prediction of distal arm posture in 3-d space from shoulder movements for control of upper limb prostheses. Proceedings of the IEEE, 96(7):1217-1225, 2008.
[18] M Farokhzadi, A Maleki, A Fallah, and S Rashidi. Online estimation of elbow joint angle using upper arm acceleration: A movement partitioning approach. J. Biomed. Phys. Eng., 2016.

[19] Manelle Merad, Agnes Roby-Brami, and Nathanael Jarrassé. Towards the implementation of natural prosthetic elbow motion using upper limb joint coordination. In Proceedings of the International Conference on Biomedical Robotics and Biomechatronics, pages 829-834, 2016.

[20] Aadeel Akhtar, Levi J Hargrove, and Timothy Bretl. Prediction of distal arm joint angles from EMG and shoulder orientation for prosthesis control. In Proceedings of the International Conference of the Engineering in Medecine and Biology Society (EMBS), pages 4160-4163, 2012.

[21] Federico Montagnani, Marco Controzzi, and Christian Cipriani. Exploiting arm posture synergies in activities of daily living to control the wrist rotation in upper limb prostheses: A feasibility study. In $E M B C$, pages $2462-2465,2015$.

[22] Nasser A Alshammary, Daniel A Bennett, and Michael Goldfarb. Efficacy of coordinating shoulder and elbow motion in a myoelectric transhumeral prosthesis in reaching tasks. In Proceedings of the International Conference on Robotics and Automation (ICRA), pages 3723-3728, 2016.

[23] Manelle Merad, Étienne de Montalivet, Agnès Roby-Brami, and Nathanaël Jarrassé. Intuitive prosthetic control using upper limb interjoint coordinations and imu-based shoulder angles measurement: A pilot study. In Proceedings of the International Conference on Intelligent Robots and Systems, pages 5677-5682. IEEE, 2016.

[24] Manelle Merad, Étienne de Montalivet, Amélie Touillet, Noël Martinet, Agnès Roby-Brami, and Nathanaël Jarrassé. Can we achieve intuitive prosthetic elbow control based on healthy upper limb motor strategies? Frontiers in Neurorobotics, 12:1, 2018.

[25] Stewe Jönsson, Kerstin Caine-Winterberger, and Rickard Brånemark. Osseointegration amputation prostheses on the upper limbs: methods, prosthetics and rehabilitation. Prosthet. Orthot. Int., 35(2):190-200, 2011.

[26] Max Ortiz-Catalan, Bo Håkansson, and Rickard Brånemark. An osseointegrated human-machine gateway for long-term sensory feedback and motor control of artificial limbs. Sci. Transl. Med., 6(257):1-7, 2014.

[27] Enzo Mastinu, Pascal Doguet, Yohan Botquin, Bo Håkansson, and Max Ortiz-Catalan. Embedded system for prosthetic control using implanted neuromuscular interfaces accessed via an osseointegrated implant. IEEE transactions on biomedical circuits and systems, 11(4):867-877, 2017.

[28] Ge et al. Wu. Isb recommendation on definitions of joint coordinate system of various joints for the reporting of human joint motion-part i: ankle, hip, and spine. Journal of Biomechanics, 2002.

[29] Freek Stulp and Olivier Sigaud. Many regression algorithms, one unified model: a review. Neural Networks, 69:60-79, 2015.

[30] Mathilde Legrand, Manelle Merad, Etienne de Montalivet, Agnès RobyBrami, and Nathanaël Jarrassé. Movement-based control for upper-limb prosthetics: Is the regression technique the key to a robust and accurate control? Frontiers in Neurorobotics, 12:41, 2018.

[31] Till Bockemühl, Nikolaus F Troje, and Volker Dürr. Inter-joint coupling and joint angle synergies of human catching movements. Hum. Mov. Sci., 29(1):73-93, 2010.

[32] Ali Hussaini, Arthur Zinck, and Peter Kyberd. Categorization of compensatory motions in transradial myoelectric prosthesis users. Prosthet. Orthot. Int., pages 1-8, 2016.

[33] Jurriaan H de Groot, Sonia M Angulo, Carel GM Meskers, Hélène CM van der Heijden-Maessen, and J Hans $\mathrm{H}$ Arendzen. Reduced elbow mobility affects the flexion or extension domain in activities of daily living. Clin. Biomech., 26:713-717, 2011.

[34] M Deijs, RM Bongers, NDM Ringeling-van Leusen, and CK van der Sluis. Flexible and static wrist units in upper limb prosthesis users: functionality scores, user satisfaction and compensatory movements. $J$. Neuroeng. Rehabil., 13(26):1-13, 2016. 